\title{
Anisotropic Initial Reaction Mechanism and Sensitivity \\ Characterization of the Layered Crystal Structure Explosive ICM- 102 Under Shock Loading
}

Kun Yang, Lang Chen*, Danyang Liu, Jianying Lu, Yiwen Xiao, Deshen Geng, Junying Wu

State Key Laboratory of Explosion Science and Technology, Beijing Institute of Technology, Beijing 100081, China

\section{Supporting Information}

\section{Shockwave front position and shockwave velocity}

The supercell was divided into 40 bins, and the average particle velocity of each bin was recorded every $10 \mathrm{fs}$. When the particle velocity increased to half of the piston speed, the shockwave is defined as the propagation to the center of the bin. The particle velocity changes as a function of time, with the bins numbered as $5,10,15,20,25$ when the shock loadings were orientated along the $x$-axis, at a speed of $2,000 \mathrm{~m} / \mathrm{s}$, is shown in Figure S1. Velocity is observed to successively increase as a function of time, and finally increases to the same speed as the piston. The intersection of the horizontal line (half of the piston speed, $1,000 \mathrm{~m} / \mathrm{s}$ ) and the particle velocity over time is the time when the shockwave propagated to the center of the bins. Hence, the propagation distance of the shockwave in the supercell could be determined.

${ }^{*}$ Corresponding author: Lang Chen.

Tel \& Fax: +86 1068914711.

E-mail: chenlang@bit.edu.cn. 


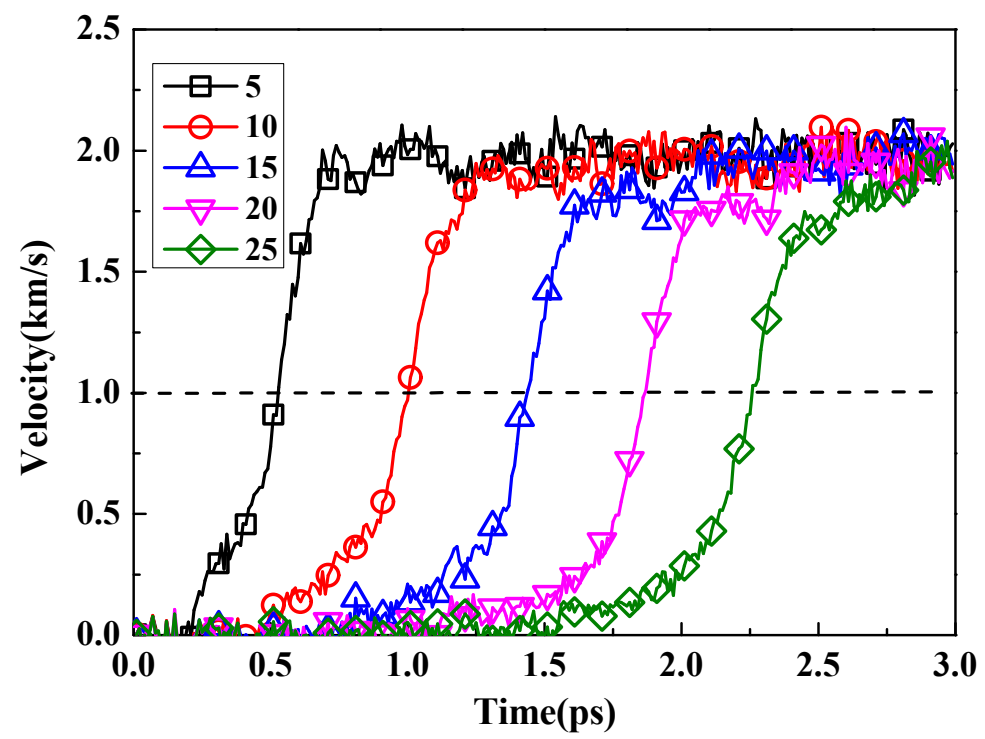

Figure S1. Particle velocity change as a function of time for bins numbered 5, 10, 15, 20, 25. The shock loadings are orientated along the $x$-axis, at a speed of $2,000 \mathrm{~m} / \mathrm{s}$.

The relationship between the propagation distance of the shockwave in the ICM102 supercell and time, when the shock loading is orientated along the $x$-axis at a speed of $2,000 \mathrm{~m} / \mathrm{s}$, is shown in Figure S2. The propagation distance of the shockwave is observed to change linearly as a function of time. Therefore, a linear fit can be performed on the relationship between the propagation distance of the shockwave and time. Additionally, the slope of the fitted line is defined as the shockwave velocity (Figure S2).

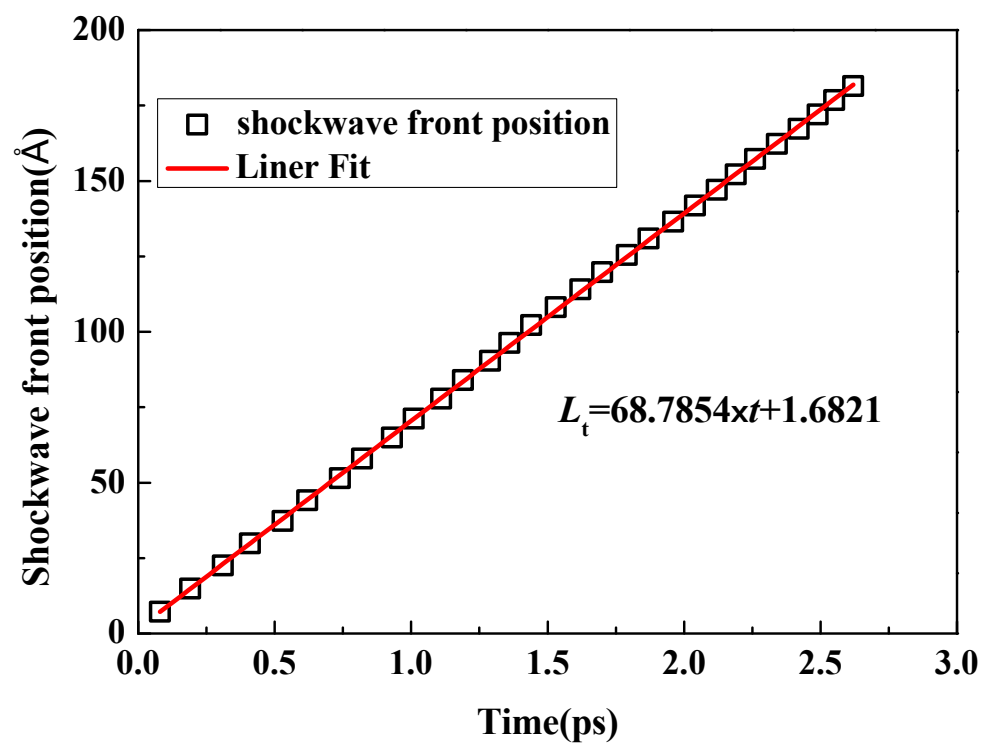

Figure S2. Relationship between the propagation distance of the shockwave in ICM-102 and time for shock loadings orientated along the $x$-axis, at a speed of $2,000 \mathrm{~m} / \mathrm{s}$. 


\section{Shockwave velocity, reaction rate and shockwave pressure}

The chemical reactions of ICM-102 under piston impact along the $x$-, $y$ - and $z$-axis for numerous speeds, ranging from $500 \mathrm{~m} / \mathrm{s}$ to $4,000 \mathrm{~m} / \mathrm{s}$, were simulated and the relationship between the reaction rate and the shockwave pressure was correlated, as shown in Table S1.

Table S1. Shockwave velocity, reaction rate and shockwave pressure as a function of shock loading orientation at various speeds.

\begin{tabular}{|c|c|c|c|c|c|c|c|c|c|c|c|}
\hline \multicolumn{4}{|c|}{ Shock loading along $x$-axis } & \multicolumn{4}{|c|}{ Shock loading along $y$-axis } & \multicolumn{4}{|c|}{ Shock loading along $z$-axis } \\
\hline $\begin{array}{c}u_{\mathrm{p}} \\
(\mathrm{m} / \mathrm{s}) \\
\end{array}$ & $\begin{array}{c}U_{\mathrm{s}} \\
(\mathrm{m} / \mathrm{s})\end{array}$ & $\delta$ & $\begin{array}{c}P \\
(\mathrm{GPa}) \\
\end{array}$ & $\begin{array}{c}\begin{array}{c}u_{\mathrm{p}} \\
(\mathrm{m} / \mathrm{s})\end{array} \\
\end{array}$ & $\begin{array}{c}\begin{array}{c}U_{\mathrm{s}} \\
(\mathrm{m} / \mathrm{s})\end{array} \\
\end{array}$ & $\delta$ & $\begin{array}{c}P \\
(\mathrm{GPa}) \\
\end{array}$ & $\begin{array}{c}u_{\mathrm{p}} \\
(\mathrm{m} / \mathrm{s}) \\
\end{array}$ & 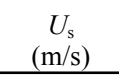 & $\delta$ & $\begin{array}{c}P \\
(\mathrm{GPa}) \\
\end{array}$ \\
\hline 500 & 3472.09 & 0.020 & 3.08 & 500 & 3957.33 & 0.025 & 3.51 & 500 & 4209.66 & 0.016 & 3.73 \\
\hline 800 & 4322.52 & 0.024 & 6.13 & 1000 & 4901.41 & 0.031 & 8.70 & 1000 & 5593.32 & 0.012 & 9.92 \\
\hline 1000 & 4917.07 & 0.012 & 8.72 & 1200 & 5312.45 & 0.015 & 11.31 & 1200 & 5850.23 & 0.026 & 12.45 \\
\hline 1100 & 5080.10 & 0.021 & 9.91 & 1400 & 5696.48 & 0.032 & 14.15 & 1400 & 6252.79 & 0.026 & 15.53 \\
\hline 1200 & 5371.17 & 0.022 & 11.43 & 1500 & 5916.50 & 0.028 & 15.74 & 1500 & 6309.43 & 0.029 & 16.79 \\
\hline 1300 & 5568.19 & 0.044 & 12.84 & 1600 & 6222.52 & 0.037 & 17.66 & 1600 & 6648.27 & 0.018 & 18.87 \\
\hline 1400 & 5841.11 & 0.056 & 14.51 & 1700 & 6346.53 & 0.058 & 19.14 & 1700 & 6855.61 & 0.043 & 20.68 \\
\hline 1500 & 5941.93 & 0.076 & 15.81 & 1800 & 6515.55 & 0.06 & 20.81 & 1800 & 7010.37 & 0.034 & 22.39 \\
\hline 1600 & 6187.75 & 0.072 & 17.56 & 1900 & 6710.56 & 0.064 & 22.62 & 1900 & 7235.92 & 0.052 & 24.39 \\
\hline 1700 & 6363.34 & 0.080 & 19.19 & 2000 & 6880.00 & 0.075 & 24.41 & 2000 & 7233.82 & 0.052 & 25.67 \\
\hline 1800 & 6549.51 & 0.104 & 20.91 & 2100 & 7077.59 & 0.081 & 26.37 & 2100 & 7545.42 & 0.062 & 28.11 \\
\hline 1900 & 6758.47 & 0.105 & 22.78 & 2200 & 7227.61 & 0.106 & 28.21 & 2200 & 7705.23 & 0.068 & 30.07 \\
\hline 2000 & 6878.54 & 0.171 & 24.41 & 2300 & 7420.62 & 0.136 & 30.28 & 2300 & 7998.55 & 0.103 & 32.64 \\
\hline 2200 & 7324.77 & 0.202 & 28.59 & 2400 & 7616.64 & 0.138 & 32.43 & 2400 & 8143.19 & 0.134 & 34.67 \\
\hline 2400 & 7627.08 & 0.308 & 32.47 & 2500 & 7768.27 & 0.209 & 34.45 & 2500 & 8227.34 & 0.166 & 36.49 \\
\hline 2500 & 7803.37 & 0.349 & 34.61 & 2600 & 8012.67 & 0.262 & 36.96 & 2600 & 8426.4 & 0.189 & 38.87 \\
\hline 2600 & 8010.38 & 0.411 & 36.95 & 2750 & 8427.71 & 0.347 & 41.11 & 2750 & 8613.52 & 0.337 & 42.02 \\
\hline 2800 & 8303.88 & 0.566 & 41.25 & 2800 & 8866.46 & 0.359 & 44.04 & 2800 & 9329.7 & 0.339 & 46.34 \\
\hline 3000 & 8624.25 & 0.693 & 45.90 & 3000 & 8676.75 & 0.496 & 46.18 & 3000 & 9036.41 & 0.478 & 48.09 \\
\hline 3200 & 8929.55 & 0.769 & 50.69 & 3200 & 8601.28 & 0.444 & 48.83 & 3200 & 9056.69 & 0.456 & 51.41 \\
\hline 3400 & 9224.64 & 0.877 & 55.64 & 3250 & 9170.77 & 0.56 & 52.87 & 3250 & 9551.13 & 0.544 & 55.07 \\
\hline 3500 & 9365.85 & 0.882 & 58.15 & 3400 & 9380.79 & 0.68 & 56.58 & 3400 & 9839.4 & 0.660 & 59.35 \\
\hline 3600 & 9469.86 & 0.927 & 60.48 & 3500 & 9472.64 & 0.808 & 58.82 & 3500 & 9951.03 & 0.799 & 61.79 \\
\hline 4000 & 10035.31 & 0.978 & 71.21 & 4000 & 10176.59 & 0.957 & 70.82 & 4000 & 10364.61 & 0.957 & 74.55 \\
\hline
\end{tabular}

\section{Density functional theory (DFT) calculation details}

In the DFT calculations, the B3LYP functional with the $6-31 \mathrm{G}^{* *}$ basis set was used, and the energy of dimerization was calculated when the length between the bonding atoms reduced from $3 \AA$ to $1 \AA$, in steps of $0.3 \AA$. As a result of the rapid speed of the molecules during the impact process, molecule deformation can be ignored over extremely short periods of time. Hence, rigid scanning was applied herein. 